\title{
A Retrospective Assessment of Mortality from the London Smog Episode of 1952: The Role of Influenza and Pollution
}

\author{
Michelle L. Bell, ${ }^{1}$ Devra L. Davis, ${ }^{2}$ and Tony Fletcher ${ }^{3}$
}

${ }^{1}$ Department of Epidemiology, Johns Hopkins Bloomberg School of Public Health, Baltimore, Maryland, USA; ${ }^{2} \mathrm{H}$. John Heinz III School of Public Policy and Management, Carnegie Mellon University, Pittsburgh, Pennsylvania, USA; ${ }^{3}$ London School of Hygiene and Tropical Medicine, London, United Kingdom

\begin{abstract}
The London smog of 1952 is one of history's most important air pollution episodes in terms of its impact on science, public perception of air pollution, and government regulation. The association between health and air pollution during the episode was evident as a strong rise in air pollution levels was immediately followed by sharp increases in mortality and morbidity. However, mortality in the months after the smog was also elevated above normal levels. An initial government report proposed the hypothesis that influenza was responsible for high mortality during these months. Estimates of the number of influenza deaths were generated using multiple methods, indicating that only a fraction of the deaths in the months after the smog could be attributable to influenza. Sensitivity analysis reveals that only an extremely severe influenza epidemic could account for the majority of the excess deaths for this time period. Such an epidemic would be on the order of twice the case-fatality rate and quadruple the incidence observed in a general medical practice during the winter of 1953 . These results underscore the need for diligence regarding extremely high air pollution that still exists in many parts of the world. Key words: air pollution, influenza, London, mortality. Environ Health Perspect 112:6-8 (2004). doi:10.1289/ehp.6539 available via $h t t p: / / d x$.doi.org/ [Online 15 October 2003]
\end{abstract}

In December 1952, a thick smog settled over London, resulting in unprecedented morbidity and mortality, bringing the relationship between air pollution and health to the attention of the general public, the government, the media, and the scientific community. The Big Smoke, a 50-year commemoration of the smog, took place in London in December 2002. The conference reviewed the events leading to the episode, the health impacts of the smog, and current air pollution conditions in London and elsewhere. This revisiting of the smog reminded us that much can still be learned from the event, in terms of both how air pollution affected Londoners at the time and how health can be affected by high levels of air pollution in much of the world today.

In an earlier study (Bell and Davis 2001), we analyzed the relationship between pollution and mortality and morbidity for the London smog of 1952. Several indicators of morbidity, such as hospital admissions, showed patterns similar to those of pollution levels. Daily mortality during the smog was also associated with daily air pollution levels. Results were not sensitive to the peak day of the pollution and were not confounded by temperature. The relationship between mortality and air pollution levels for longer periods revealed a statistically significant association between weekly ambient levels and mortality. Regression analysis showed that weekly air pollution and mortality were statistically associated, even when the week of the episode was removed from the data set, leaving only air pollution concentrations far below the episode (Bell and Davis 2001).
Thus, air pollution was affecting mortality in London, aside from the extreme episode.

Mortality did not return to normal levels for several months after the episode. The strong immediate health response to the episode is evident in the coinciding sharp increase in mortality; however, the elevated mortality in the months after the smog requires a more detailed analysis. An initial government report (U.K. Ministry of Health 1954) proposed the hypothesis that influenza was responsible for the elevated mortality in the months that followed the episode. The weekly number of excess deaths in Greater London (the number of deaths exceeding those during the same time period the previous year) peaked at about 4,500 for the week ending 13 December 1952 . Total mortality rates were about $80 \%$ higher than the previous year for December 1952 and were 50 and 40\% higher, respectively, for January and February 1953. From December 1952 through March 1953, there were over 13,500 more deaths than normal. A fraction of these likely resulted from air pollution and a fraction from influenza.

The exact numbers of influenza-related and air pollution-related deaths are unknown and continue to generate debate (Stone 2002). Estimates of influenza deaths generated through multiple approaches contradict the influenza theory, indicating that far more people died from air pollution than originally believed. Estimates of the number of influenza deaths were constructed using observations from general practice of medicine in the London area and the control group of a vaccine study conducted at the time. The reconstructed estimates of influenza mortality reveal that only a portion of the excess deaths after the smog can be attributed to influenza.

Here we expand on previous analysis (Bell and Davis 2001) by providing additional estimates of the number of influenza-related deaths and by exploring how extensive an influenza epidemic would have to be to account for the excess mortality. The following describes a sensitivity analysis that explores how high influenza incidence and case-fatality rates would have to be to account for the increased mortality, indicating that such an explanation is highly unlikely.

\section{Estimates of Influenza-Related Mortality}

The number of deaths from influenza was estimated using two approaches: observations from a general medical practice and data from a vaccine trial. Observations from a general medical practice of about 6,000 persons in Greater London from 1949 through 1968 provided an estimated case-fatality rate of $0.2 \%$ (Fry 1969). For the years that had an influenza epidemic, Fry estimated that the percentage of patients who contracted influenza ranged from $3 \%$ to $17 \%$ and averaged $8 \%$ with a median of $6.5 \%$. For the 1953 year, $6 \%$ of the patients had influenza. This supports other documents that reported a mild influenza epidemic during that time (U.K. Ministry of Health 1953, 1956).

The Medical Research Council Committee on Clinical Trials of Influenza Vaccine organized a clinical trial of an influenza vaccine with 12,710 volunteers in London and other cities (Committee on Clinical Trials of Influenza Vaccine 1953). The majority of volunteers were inoculated between 26 November and 5 December 1952, with some inoculations on 12 December 1952. Follow-up continued

Address correspondence to M. Bell, Department of Epidemiology, Johns Hopkins Bloomberg School of Public Health, 615 North Wolfe Street, W6508-A, Baltimore, MD 21205 USA. Telephone: (410) 6143261. Fax: (410) 955-0863. E-mail: mbell6@jhu.edu

We thank H. Ellis, R. Maynard, D. Bates, and R. Le Bruin for their assistance. We also thank all participants of the Big Smoke, a 50-year commemoration of the smog, which took place in London in December 2002.

The authors declare they have no competing financial interests.

Received 19 June 2003; accepted 15 October 2003. 
through 31 March 1953. The attack rate in the control group was $4.9 \%$ during the winter of 1952-1953. The original researchers divided areas into three regions based on their mortality rates because high mortality areas were assumed to have higher incidence of influenza. London was in the highest incidence category, with an attack rate of $6.61 \%$ in the control group of 1,181 volunteers. This value is similar to the incidence rate observed for the winter of 1953 for the general medical practice.

The original government report on the health effects of the episode (U.K. Ministry of Health 1954) proposed that an influenza epidemic caused the elevated mortality, although those authors recognized that some deaths in the months after the smog could be related to the air pollution from the episode. Excess deaths from this time through the end of March number more than 8,000 . The report provides estimates of the number of deaths attributable to the smog using several different approaches, which do not include any deaths after 20 December 1952. The U.K. Ministry of Health estimated that 5,655 people died from influenza in the first 3 months of 1953 (U.K. Ministry of Health 1956). Another source reported 5,647 influenza deaths for the first 3 months of 1953 in England and Wales (World Health Organization 1953).

To better understand the role of influenza and pollution in the deaths that took place in the months after the extreme episode, an estimate of the number of influenza-related deaths was constructed using information from the observations of a general medical practice and the vaccine trial. The population of Greater London at this time was approximately 8.6 million, based on the 1952 census (U.K. General Register Office 1951). Because early government reports attributed no deaths after 20 December 1952 to pollution and because mortality rates remained elevated for several months, especially for January and February 1953, we calculated the number of excess deaths from 21 December 1952 through 28 February 1953. During this period, 8,275 more deaths occurred than expected based on the previous year, which did not have an influenza epidemic or extreme air pollution events.

Figure 1 shows the fraction of deaths from 21 December 1952 through February 1953 that are related to influenza using four approaches; the original government estimate for January through March 1953 (Committee on Clinical Trials of Influenza Vaccine 1953, Fry 1969, U.K. Ministry of Health 1953) is provided for comparison. The reconstructed estimates are conservative in several ways.

All yearly influenza deaths from the observations from a general medical practice, attack rates from December through March 1953 from the vaccine trial, and the government estimate for January through March 1953 were applied to the shorter period of less than 2.5 months (Committee on Clinical Trials of Influenza Vaccine 1953, Fry 1969, U.K. Ministry of Health 1953). The government estimate was based on all deaths in England, yet in Figure 1 is compared to estimates only for Greater London. The reconstructed estimates indicate that about 1,000-1,400 influenza-related deaths took place during this period. The government estimate also leaves more than 2,600 deaths unexplained. Under each of these approaches, a significant portion of the excess mortality is unexplained by the influenza theory.

\section{Sensitivity Analysis}

We performed a simple sensitivity analysis to explore how different values for the incidence or case-fatality rate could influence influenza mortality estimates and the fraction of unexplained deaths. This is important given that the exact number of influenza deaths is unknown and the approaches used here could conceivably underestimate or overestimate the actual number of deaths attributable to influenza. For example, the incidence rates generated through observations in a general medical practice are likely to be lower than the true incidence because some patients with influenza may not have consulted their physician. It is plausible that the underestimation is large if a significant number of patients with influenza did not consult their primary physician and he did not learn of their condition. In contrast, the case-fatality rate could be an overestimation, assuming the most ill patients sought medical care and few of the patients with influenza that did not receive medical care died. The attack rates from the vaccine control study (Committee on Clinical Trials

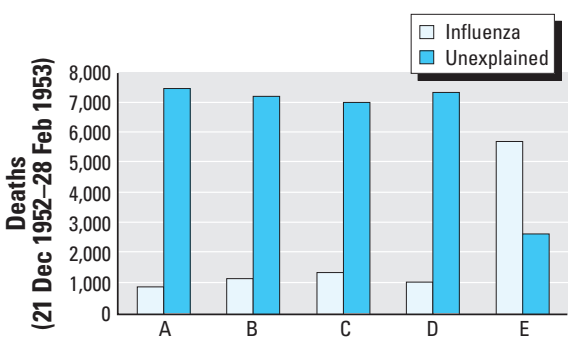

Figure 1. Estimated number of influenza deaths in Greater London, 21 December 1952 through 28 February 1953. Methods of estimation are as follows: $A$, control group from influenza vaccine study; $B$, control group from influenza vaccine study, for areas of high mortality; $C$, observations from a general medical practice, using the average incidence rate of years with influenza epidemics; $D$, observations from a general medical practice, using the incidence rate for the 1953 winter; $E$, government estimate for January to through March 1953. Data from the Committee on Clinical Trials of Influenza Vaccine (1953), Fry (1969), and the U.K. Ministry of Health (1953). of Influenza Vaccine 1953) could be lower than those in the general population because of the selection process of volunteers for the study.

Because of these possibilities, we performed a sensitivity analysis by increasing both the incidence and case-fatality rates. Figure 2 provides the number of influenza deaths from 21 December 1952 through 28 February 1953 if the estimate generated using the 1953 winter rate from the general medical practice is too low by varying degrees. Results show that the estimated case-fatality and incidence rates for influenza would have to be drastically understated for influenza to account for the excess mortality after the smog. The most severe epidemic observed during the 20-year period of the general medical practice observations took place in 1957. If influenza after the pollution episode were twice that severe, many deaths still remain unexplained. If both the 1953 incidence rate and the case-fatality rate were underestimated by $100 \%$, the corrected value of influenza deaths would be approximately 4,000. Only an extremely severe influenza epidemic could account for the majority of the excess deaths for this period. Such an epidemic would be along the order of twice the normal case-fatality rate and quadruple the incidence observed in 1953. These results reveal that even if the estimates of the number of influenza-related deaths, as presented in Figure 1, are substantially lower than the true values, a larger number of deaths remain unexplained by influenza.

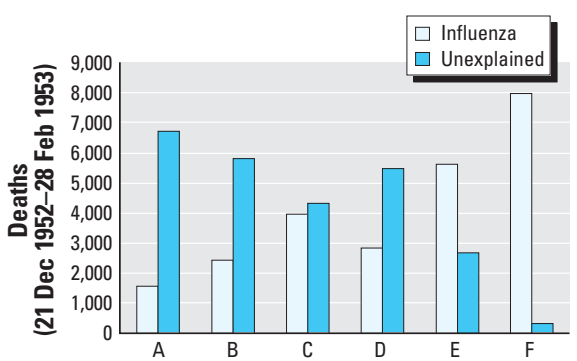

Figure 2. Sensitivity analysis for number of influenza deaths in Greater London, 21 December 1952 through 28 February 1953. Methods of sensitivity analysis estimates are as follows: $A$, casefatality rate and incidence from the observations of a general medical practice for the 1953 winter both increased by $25 \%$; $B$, case-fatality rate and incidence from the observations of a general medical practice for the 1953 winter both increased by $50 \%$; $C$, case-fatality rate and incidence from the observations of a general medical practice for the 1953 winter both increased by $100 \% ; D$, incidence of the most severe influenza epidemic observed for the general medical practice from 1949 through 1968 (17\% for 1957 epidemic); $E$, incidence twice that of the 1957 epidemic $(34 \%)$; $F$, case-fatality doubled and incidence four times higher than from the observations of a general medical practice for the 1953 winter. Data from Fry (1969). 


\section{Discussion}

Our analysis shows that only a fraction of the elevated mortality in the months after the 1952 London smog can be attributed to influenza, leaving thousands of deaths otherwise unexplained. Sensitivity analysis illustrates that an influenza epidemic of enormous proportions would be required to explain the excess mortality. Such an epidemic would have to be about three times larger than the most severe epidemic recorded from 1949 to 1968.

These excess deaths could be attributable to air pollution through a delayed effect from the smog itself. They could also be related to air pollution concentrations that remained above normal levels through January 1953. Mortality in London was affected by air pollution during these years aside from the extreme episode. Regression analysis of weekly pollution and mortality levels from October 1952 through March 1953 and the corresponding weeks of the previous year (October 1951 through March 1952), controlling for temperature, found a statistically significant association, even when the week of the episode was omitted (Bell and Davis 2001). If the excess deaths in the months after the 1952 London smog are related to air pollution, the mortality count would be approximately 12,000 rather than the 3,000-4,000 generally reported for the episode. Additionally, there could be interaction between influenza and air pollution, in that people who survived the extreme episode could have been more susceptible to influenza.

A recent analysis of lung tissues from persons who died during the smog episode found soot and other particle types in the lung (Hunt et al. 2003). The particulate matter (PM) was an aggregation of ultrafine PM (PM with an aerodynamic diameter $\leq 0.1 \mu \mathrm{m}$ ) and PM with a diameter $<1 \mu \mathrm{m}$. Carbonaceous PM was found in each compartment of the lung; however, heavy-metal-bearing particles such as lead were identified in the compartments of the lung representing recent exposure and were not found in compartments indicative of longer term exposure.
A review of limited autopsy records available from the Royal London Hospital reported twice as many deaths with chronic obstructive pulmonary disease (COPD) as a major finding from December 1952 through February 1953 as corresponding months in other years (Hunt et al. 2003). COPD mortality has been linked with exposure to air pollution (Karakatsani et al. 2003; Sunyer and Basagana 2001); thus, the autopsy reports support the hypothesis that air-pollution-related deaths continued after the peak episode.

These new lessons learned from the study of the 1952 London smog indicate that the episode had a much larger impact on health than previously reported. Conditions as extreme as the 1952 London smog are unlikely in industrialized nations today; however, London's air pollution still affects human health. For instance, deaths and hospital admissions during the week of an air pollution episode in December 1991 had higher mortality rates and hospital admissions than during the week before the episode and during previous time periods (Anderson et al. 1995). A study of air pollution in London from April 1987 through March 1992, which accounted for influenza, identified associations between daily mortality and concentrations of black smoke and ozone (Anderson et al. 1996).

Exceedingly high air pollution still exists in many parts of the world and causes a substantial health burden. Ezzati et al. (2002) estimated that almost 800,000 deaths are caused each year by urban outdoor air pollution and more than 1.6 million deaths annually from indoor air pollution. Additionally, health effects have been observed at low pollution levels (Vedal et al. 2003). The relationship between air pollution and mortality has been demonstrated in numerous locations using increasingly sophisticated statistical methodology and a variety of study designs (Katsouyanni et al. 1997; Samet et al. 2000). The analysis presented here underscores the need to address modern-day air pollution problems.

\section{REFERENCES}

Anderson HF, Limb ES, Bland JM, deLeon AP, Strachan DP, Bower JS. 1995. Health effects of an air pollution episode in London, December 1991. Thorax 50:1188-1193.

Anderson HR, deLeon AP, Bland M, Bower JS, Strachan DP. 1996. Air pollution and daily mortality in London: 1987-1992. Br Med J 312:665-669.

Bell ML, Davis DL. 2001. Reassessment of the lethal London fog of 1952: novel indicators of acute and chronic consequences of acute exposure to air pollution. Environ Health Perspect 109(suppl 3):389-394.

Committee on Clinical Trials of Influenza Vaccine. 1953. Clinical trials of influenza vaccine: a progress report to the Medical Research Council by its Committee on Clinical Trials of Influenza Vaccine. Br Med J 4847:1173-1177.

Ezzati M, Lopez AD, Rodgers A, Vander Hoorn S, Murray CJL, Comparative Risk Assessment Collaborating Group. 2002 Selected major risk factors and global and regional burden of disease. Lancet 360:1347-1360.

Fry J. 1969. Epidemic influenza. Patterns over 20 years (19491968). J R Coll Gen Pract 17:100-103.

Hunt A, Abraham JL, Judson B, Berry CL. 2003. Toxicologic and epidemiologic clues from the characterization of the 1952 London smog fine particulate matter in archival autopsy lung tisses. Environ Health Perspect 111:1209-1214.

Karakatsani A, Andreadaki S, Katsouyanni K, Dimitroulis I, Trichopoulos D, Benetou V, et al. 2003. Air pollution in relation to manifestations of chronic pulmonary disease: a nested case-control study in Athens, Greece. Eur J Epidemiol 18:45-53.

Katsouyanni K, Touloumi G, Spix C, Schwartz J, Balducci F, Medina S, et al. 1997. Short-term effects of ambient sulphur dioxide and particulate matter on mortality in 12 European cities: results from time series data from the APHEA project. Air pollution and health: a European approach. Br Med J 314:1658-1663.

Samet JM, Zeger SL, Dominici F, Curriero F, Coursac I, Dockery DW, et al. 2000. The National Morbidity, Mortality, and Air Pollution Study Part II: Morbidity and Mortality from Air Pollution in the United States. Boston, MA:Heath Effects Institute.

Stone R. 2002. Counting the cost of London's killer smog Science 298:2106-2107.

Sunyer J, Basagana X. 2001. Particles, and not gases, are associated with the risk of death in patients with chronic obstructive pulmonary disease. Int J Epidemiol 30:1138-1140.

U.K. General Register Office. 1951. Census 1951, England and Wales. London:Her Majesty's Stationery Office.

U.K. Ministry of Health. 1953. The Report of the Chief Medica Officer on the State of Public Health. London:Her Majesty's Stationery Office.

- 1954. Mortality and Morbidity during the London Fog of December 1952. Reports on Public Health and Medical Subjects No. 95. London:Ministry of Health.

. 1956. The Report of the Chief Medical Officer on the State of Public Health. London:Her Majesty's Stationary Office.

Vedal S, Brauer M, White R, Petkau J. 2003. Air pollution and daily mortality in a city with low levels of pollution. Environ Health Perspect 111:45-51.

World Health Organization. 1953. 1952/53 influenza epidemic in the Northern Hemisphere. Epidemiol Vital Stat Rep 6:203-226. 